\title{
EchoGéo
}

11 | 2010

Madagascar

\section{Gémellité du deuil et de l'espoir}

Jean Marie Théodat

\section{(2) OpenEdition}

Journals

Édition électronique

URL : https://journals.openedition.org/echogeo/11729

DOI : 10.4000/echogeo.11729

ISSN : 1963-1197

\section{Éditeur}

Pôle de recherche pour l'organisation et la diffusion de l'information géographique (CNRS UMR 8586)

Référence électronique

Jean Marie Théodat, "Gémellité du deuil et de l'espoir », EchoGéo [En ligne], 11 | 2010, mis en ligne le 24 février 2010, consulté le 31 juillet 2021. URL : http://journals.openedition.org/echogeo/11729 ;

DOI : https://doi.org/10.4000/echogeo.11729

Ce document a été généré automatiquement le 31 juillet 2021.

EchoGéo est mis à disposition selon les termes de la licence Creative Commons Attribution - Pas d'Utilisation Commerciale - Pas de Modification 4.0 International (CC BY-NC-ND) 


\title{
Gémellité du deuil et de l'espoir
}

\author{
Jean Marie Théodat
}

1 S'il est normal que l'actualité éditoriale d'une revue reflète les préoccupations de l'époque, il est rare que le sommaire d'un numéro rappelle avec autant d'insistance les aléas géographiques d'une identité nationale qui, pour être sans cesse invoquée, remise en exergue par les politiques, n'en continue pas moins d'être remise en question par les événements qui marquent notre quotidien. Dans sa onzième livraison, la revue EchoGéo aborde des territoires et des thèmes dont le point commun est d'avoir participé à donner de la France une idée géométriquement plus vaste que l'hexagone où se trouve confinée aujourd'hui la représentation nationale.

2 Cette "plus grande France" d'hier se rappelle à notre souvenir par le biais de la catastrophe qui vient de frapper la République d'Haïti, pays dont la geste révolutionnaire est dans une relation de gémellité historique avec la nôtre. Jean Marie Théodat rappelle, dans la rubrique Sur le Vif, les leçons à retenir du séisme qui a frappé la capitale haïtienne, en insistant sur la double dimension internationale et nationale des inflexions révélées par la catastrophe. Pour être nées d'un même élan révolutionnaire, les relations entre les deux Républiques de France et d'Haïti se sont distendues au point qu'il a fallu attendre deux cent six ans pour qu'un président de la République Française se rende pour la première fois à Port-au-Prince. Dans la tragédie se révèle une empathie fondée sur une connivence culturelle et linguistique que l'inimitié entre les gouvernements successifs et les soubresauts des relations internationales n'ont guère entamée. L'immensité du deuil n'a d'égale que l'espoir de relations plus chaleureuses suscité par la visite historique de Nicolas Sarkozy à Port-auPrince le 17 février 2010.

3 Il est intéressant de constater que les retrouvailles dans la douleur entre la France et Haïti se scellent sur fond d'interrogation référendaire dans les Départements Français d'Amérique. Appelés à se prononcer sur l'avenir du lien avec la métropole, les Martiniquais et les Guyanais ont répondu négativement à la question de savoir si ils voulaient davantage d'autonomie. Ce résultat sonne comme le glas des tendances indépendantistes et le désaveu des élites locales qui réclamaient plus de marge de manœuvre dans la gestion des affaires locales. Cependant, le résultat du double vote 
martiniquais et guyanais a été largement occulté par la tragédie haïtienne. Il s'agit pourtant, à bien y regarder, d'identités voisines qui auraient pu se trouver du même côté de l'hexagone si les circonstances historiques et la révolte des esclaves en 1791 n'en avaient pas décidé autrement. L'indépendance haïtienne a coupé les ponts entre la Saint-Domingue coloniale et les autres Antilles françaises, mais à travers le séisme et le référendum, c'est la même identité française qui se trouve sollicitée et réactivée dans le sens de l'affirmation d'un attachement renouvelé, à l'ensemble français pour les DFA, retrouvé à l'ensemble francophone, pour Haïti. La rubrique Sur le Champ, avec des contributions de Christian Jost sur la Nouvelle-Calédonie, de Jean-Marc Régnault et de Fred Constant, rappelle l'actualité de ce lien historique qui n'en finit pas de se remodeler à mesure que l'identité d'outre-mer évolue sous le double effet des luttes sociales et des représentations collectives.

Dans cette même rubrique, nous revenons sur une autre île, Madagascar, qui fut également colonie française, et qui prit son indépendance en 1958. Les contributions de Sophie Moreau, de Daniel Peyrusaubes, de Landry Ramarojohn, de Rivo Andrianirina et d'André Teyssier, rappellent le long chemin parcouru par la Grande Ile, depuis l'indépendance, et son inscription dans la mondialisation. L'ouverture sur le systèmemonde y prend parfois l'aspect d'une mise à l'encan des ressources foncières et forestières d'un pays dont l'inscription dans la mondialisation se fait au mépris des grands équilibres environnementaux et sociaux, au risque de la déstabilisation politique. La vulnérabilité des pays les moins avancés dans la compétition mondiale est à la fois économique et environnementale.

5 La catastrophe qui a frappé la capitale haïtienne révèle en creux la fragilité des systèmes urbains des pays du Sud qui font face à une croissance démographique et une transition urbaines souvent marquées par des dysfonctionnements majeurs. Mais Haïti n'est pas seul à faire face à de tels défis. D'autres pays sont confrontés à une urbanisation sans frein et sans plan dont les effets, aux limites de la zone urbanisée, dénotent des processus singuliers qui ne participent plus d'une logique rurale sans être tout à fait réductibles à l'urbanisation classique. La rubrique Sur le Terrain, à travers les contributions de Cécile Faliès, et de Cécilia Montoya, sur deux métropoles latinoaméricaines, Lima et Santiago du Chili, de Julie Le Gall et de Matìas Garcìa, sur les périphéries de Buenos Aires, de Gwenaëlle Raton enfin, sur les périphéries de Bamako, permet de prendre la mesure des recompositions spatiales à l'œuvre dans les périphéries des métropoles urbaines.

6 Le mode d'intervention des pouvoirs publics français, souvent décrié comme la manifestation d'un jacobinisme centralisateur, a pourtant trouvé son contrepoint et une vocation nouvelle dans l'inspiration des programmes européens d'aménagement du territoire. La rubrique Sur le Métier est l'occasion de donner la parole à deux géographes français dont le savoir-faire a servi de cadre de référence cartographique et thématique aux instances de décisions européennes. En effet, Alexis Sierra a réalisé deux entretiens dans lesquels Jean-François Drevet et Jean-Pierre Palisse, géographes " engagés » dans les politiques d'aménagement urbain et régional de l'Europe. Ils livrent ici leur analyse du rôle décisif de la discipline dans la gouvernance intégrée des nouvelles entités européennes, saisies comme un bloc articulé en instances emboîtées.

7 La rubrique Sur l'Ecrit rappelle l'actualité éditoriale ayant trait aux articles du Champ et les nouvelles acquisitions de la Bibliothèque de géographie. Enfin, la rubrique Sur l'Image met en lumière la double implication de sens que suppose le support 
iconographique dans l'appréhension du savoir par le terrain. L'article d'Elsa Bignante permet de mieux comprendre la démarche des Maasai et le mode de constitution des savoirs associés à leur culture, à travers l'image. 